\title{
The long-term variability of BL Lac object PKS $0735+178$
}

\author{
J.H. Fan ${ }^{1-3}$, G.Z. Xie ${ }^{4}$, R.G. Lin ${ }^{2}$, Y.P. Qin ${ }^{4}$, K.H. Li ${ }^{4}$, and X. Zhang ${ }^{4,5}$ \\ 1 CCAST(World Laboratory), P.O. BOX 8730, Beijing, China \\ 2 Center for Astrophysics, Guangzhou Normal University, Guangzhou 510400, China \\ 3 Joint Laboratory for Optical Astronomy, Chinese Academy of Sciences, China \\ ${ }^{4}$ Yunnan Observatory, Chinese Academy of Sciences, China \\ 5 Department of Physics, Yunnan Normal University, Kunming, China
}

Received June 14, 1996; accepted January 31, 1997

\begin{abstract}
The long-term variability of PKS $0735+178$ in $B$ band is shown with some new data presented and discussed by means of Jurkevich's method. The results show with good possibility the presence of a period of 14.2 years. The period of 4.89 years found by Webb et al. (1988) and Smith et al. (1987) might be confirmed with more observations, and their period of 1.2 years can be ruled out.
\end{abstract}

Key words: BL Lac objects: general - 0735+178: individual

\section{Introduction}

In active galactic nuclei (AGNs) at least two types of variability may be present, i.e. the short-term and the long-term variations. The short-term variation is probably non-periodic although a short-term period has been indeed observed in our monitoring programme of BL Lac objects (Xie et al. 1988, 1990), the long-term variation has in some cases claimed to be periodic. For example, the 11.4 years period which Webb et al. (1988) found in the light curve of 3c 345 successfully predicted the 1991 outburst of the object (Kidger \& Takalo 1990), the 12 years period found in BL Lac object OJ 287 (Sillanpaa et al. 1988; Kidger et al. 1992) also successfully predicted the optical outburst in the fall of 1994 of the object (Sillanpaa et al. 1996). To establish the reality of such long periods two prerequisites must be satisfied. First, the data sample must be of very long duration: at least six times the length of the periodicity being claimed would seem to be a valid criterion for demonstrating that any possible period is not simply a random event and probably has some physical significance. Secondly, the possible period should be of large amplitude and thus directly visible in the light curve; where

Send offprint requests to: J.H. Fan the amplitude is low, many more than six cycles of the period will be necessary to demonstrate the reality. Some AGNs seem to satisfy these two prerequisites and have shown period variations (ON 231 Liu et al. 1995; OJ 287, Sillanpaa et al. 1988a; Kidger et al. 1992; Sillanpaa et al. 1996).

PKS $0735+178$ has been classified as a BL Lac object by Carswell et al. (1974). Its measured redshift is based on a simple absorption doublet of Mg II (Burbidge \& Hewitt 1987). Observations show it has a flat radio spectrum and radio bursts (Ulvestad \& Johnston 1984; Antonucci \& Ulvestad 1985; Baath \& Zhang 1990; Zhang et al. 1991; Terasranta et al. 1991, 1992; Stevens et al. 1994), violent optical variations (Pollock et al. 1979; Worrall et al. 1981; Miller 1983; Xie et al. 1992), high polarization and superluminal motion (Aller et al. 1985; Wardle \& Roberts 1988; Porcas 1987; Baath \& Zhang 1991; Briadle et al. 1986; Impey et al. 1987): $\beta_{\text {app }}=2.8 c-7.9 c, P_{\text {rad }}=4.1-7.6 \%$, $P_{\mathrm{IR}}=12.1-16.5 \%, P_{\text {opt. }}=35 \%$. Bregman et al. (1984) also noticed that its infrared polarization is associated with flux. Webb et al. (1988) and Smith et al. (1987) constructed its historical optical light curve, searched for its periodicity using the Fourier analysis and found periods of 1.2 and 4.89 years. From the paper of Kidger et al. (1992), we have that the Fourier analysis is not as good as the Jurkevich's method to find periodicities. So, we have used the powerful method of Jurkevich (Jurkevich 1971; Kidger et al. 1992) to determine the periodicity of PKS 0735+178.

In this paper, we mainly show the optical variability with some new observations presented and discuss its periods by means of the method (Jurkevich 1971). The paper has been arranged as follows: In Sect. 2 we give the Jurkevich method, in Sect. 3 the observation data including our new data, and in Sect. 4 discussion and results.

\section{Jurkevich method}

The Jurkevich method is based on the expected mean square deviation and it is less inclined to generate 
spurious periodicities than Fourier analysis. It tests a run of trial periods around which the data are folded. All data are assigned to $m$ group according to their phases around each trial period and the variance $V_{i}^{2}$ for each group, and the sums $V_{m}^{2}$ of all groups are computed. For a trial period equal to the true one, $V_{m}^{2}$ reaches its minimum, and a "good" period will give a much reduced variance relative to those given by other false trial periods and with almost constant values. Kidger et al. (1992) introduced a fraction of the variance

$f=\frac{1-V_{m}^{2}}{V_{m}^{2}}$

where $V_{m}^{2}$ is the normalized value. In the normalized plot, a value of $V_{m}^{2}=1$ means $f=0$ and hence there is no periodicity at all. The best periods can be identified from the plot: a value of $f \geq=0.5$ suggests there is a very strong periodicity and a value of $f<0.25$ suggests that the periodicity, if genuine, is a weak one.

\section{Observation data}

PKS 0735+178 has been observed for 90 years back to JD 2417233. Optical observations indicate that not only does it have large variation with long term scale $(B=13.90-17.22)$ (Pollock et al. 1979) but also it has a short-time scale variation: $\Delta B=0.5 / 1$ weak (McGimsey et al. 1975); $\Delta m=0.49 / 1$ day (Miller 1983) and $\Delta m=0.68 / 24 \mathrm{~min}$ (Xie et al. 1992). Our group have also monitored the object since 1982 (Xie et al. 1987, 1988, 1992, 1994). During December 1994, January 1995, and January and February 1996, it was observed with the $102 \mathrm{~cm}$ RCC telescope at Yunnan Observatory and the $156 \mathrm{~cm}$ RCC telescope at Shanghai Observatory, which were equipped with direct CCD cameras. The daily averaged magnitudes are given in Table 1. All magnitudes observed from it will be published in a separate paper altogether with other BL Lac objects observed during the period (Xie et al. 1997). From our recent observation, PKS 0735+178 variated in a range of 16.89 (JD 2449691.65)-15.77 (JD 2450107.5) magnitudes in $B$ band. In this paper the historic optical data in $B$ band (434 points) are from the literatures (Wing 1973; Carswell et al. 1974; Pollock 1975; O'Dell et al. 1978; Zekl et al. 1981; Moles et al. 1985; Smith et al. 1987b; Webb et al. 1988; Sillanpaa et al. 1988b; Tanzi 1989; Takalo 1991, 1992; Valtaoja et al. 1993) with the $m_{\mathrm{pg}}$ in the paper of Zekl et al. (1981) changed into $B$ by $B-m_{\mathrm{pg}}=0.28$ (Kidger $1989 ; \mathrm{Lu} 1972$ ). Also those observed by our group (Xie et al. 1987, 1988, 1992, 1994) and those in the present paper (Table 1) are shown in Fig. 1. Observation indicate that there was a large variation of about 4.6 magnitudes during JD $2400 \quad 000+43200$ and 44200. From the $U B V R I$ data in the literatures we have $B-V=0.545 \pm 0.158$ (67 pairs data), $B-U=0.58 \pm 0.104$
(54), $B-R=0.993 \pm 0.149(55), B-I=1.551 \pm 0.218$

(55), the errors are the 1 sigma dispersion of the data.

Table 1. CCD photometry of PKS $0735+178$

\begin{tabular}{ccc}
\hline $\begin{array}{c}\text { UT Date } \\
\text { day/mon/yr }\end{array}$ & $\begin{array}{c}B \\
\text { Mag. }\end{array}$ & $\begin{array}{c}V \\
\text { Mag. }\end{array}$ \\
\hline $04 / 12 / 94$ & $16.89 \pm 0.10$ & $15.92 \pm 0.08$ \\
$05 / 12 / 94$ & $16.90 \pm 0.10$ & $16.02 \pm 0.08$ \\
$06 / 12 / 94$ & $16.85 \pm 0.10$ & $15.97 \pm 0.08$ \\
$23 / 01 / 95$ & $16.03 \pm 0.14$ & $15.30 \pm 0.06$ \\
$25 / 01 / 95$ & $16.46 \pm 0.08$ & \\
$26 / 01 / 95$ & $16.01 \pm 0.05$ & $15.35 \pm 0.09$ \\
$27 / 01 / 95$ & $16.02 \pm 0.08$ & $15.50 \pm 0.06$ \\
$28 / 01 / 95$ & $15.89 \pm 0.08$ & $15.46 \pm 0.04$ \\
$29 / 01 / 95$ & $15.96 \pm 0.09$ & $15.48 \pm 0.14$ \\
$24 / 01 / 96$ & $15.77 \pm 0.08$ & \\
$15 / 02 / 96$ & \multicolumn{2}{|c}{$15.60 \pm 0.04$} \\
\hline
\end{tabular}

To counteract the heavy weighting of the recent data, we have calculated 10-day means of the light curve. This interval is short enough compared to the long-term periods (years) and thus unlikely to distort the long-term variations too much. This light curve consists of 218 points and is shown in Fig. 2. When the method in Sect. 2 is used to the averaged data $(m=10)$, two strong periods: 14.2 years $\left(V_{m}^{2}=0.637, f=0.570\right)$; 28.69 years $\left(V_{m}^{2}=0.599, f=0.669\right)$ have been found(see Fig. 3 for detail). The former is about half the latter, and they are in fact the same with the latter being just a harmonic of the former.

\section{Discussion}

If the period of 14.2 years is really the true period of variation, taking the first relative maximum of the light curve (JD 2418346) as the first outburst, we can obtain the expected following outbursts and indicate them in vertical light line in Fig. 1. It seems that the expected outbursts are well consistent with the observation. Also, we can say two outbursts may have not been observed since there are no available data during the periods of JD $2400000+22375$ and 25645 , and JD $2400000+36227$ and 41279 . Webb et al. (1988) and Smith et al. (1987) constructed the light curve of PKS $0735+178$ (JD 2400000+40896 and 46520), analysed its period and found periods of 4.89 and 1.2 years. From Fig. 3 it can be seen that there is a very weak period of 4.85 years $\left(V_{m}^{2}=0.80, f=0.25\right)$ and there is no period of 1.2 years. When we consider the data during the period of 1970 and 1996 , we found that the period of 4.89 years is only a possible period $\left(V_{m}^{2}=0.70, f=0.428\right)$. Since the data in the considered 90 years are not so well distributed as there are no data during period of JD $2400000+22375$ and 25645, and JD 2400000+36227 and 41279 and there 


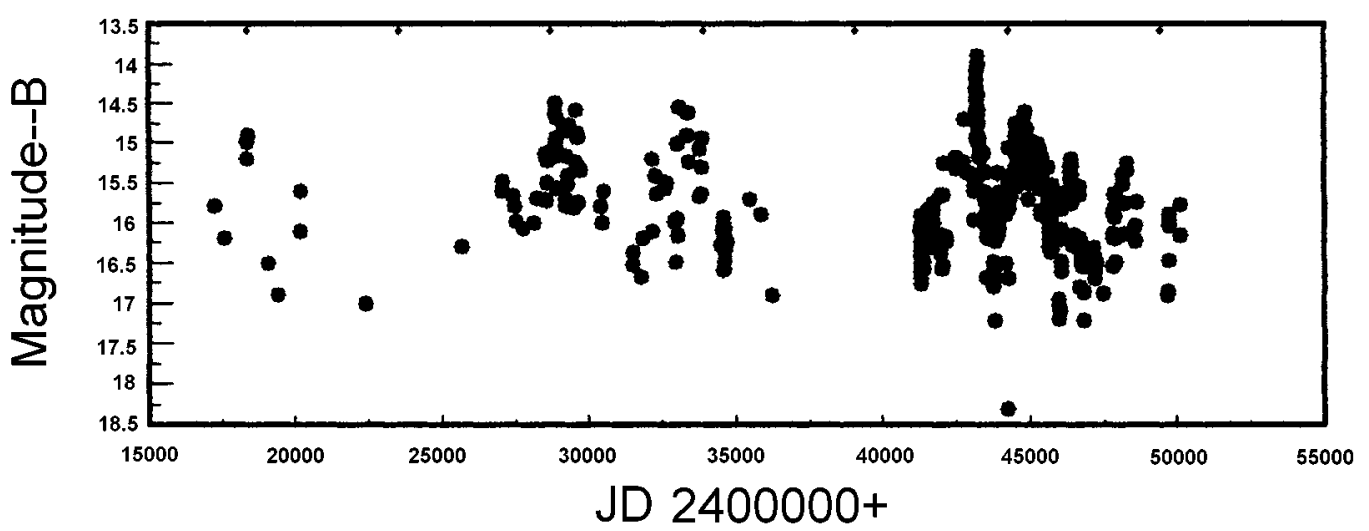

Fig. 1. The light curve in $B$ band of PKS $0735+178$ for about 90 years (434 points)

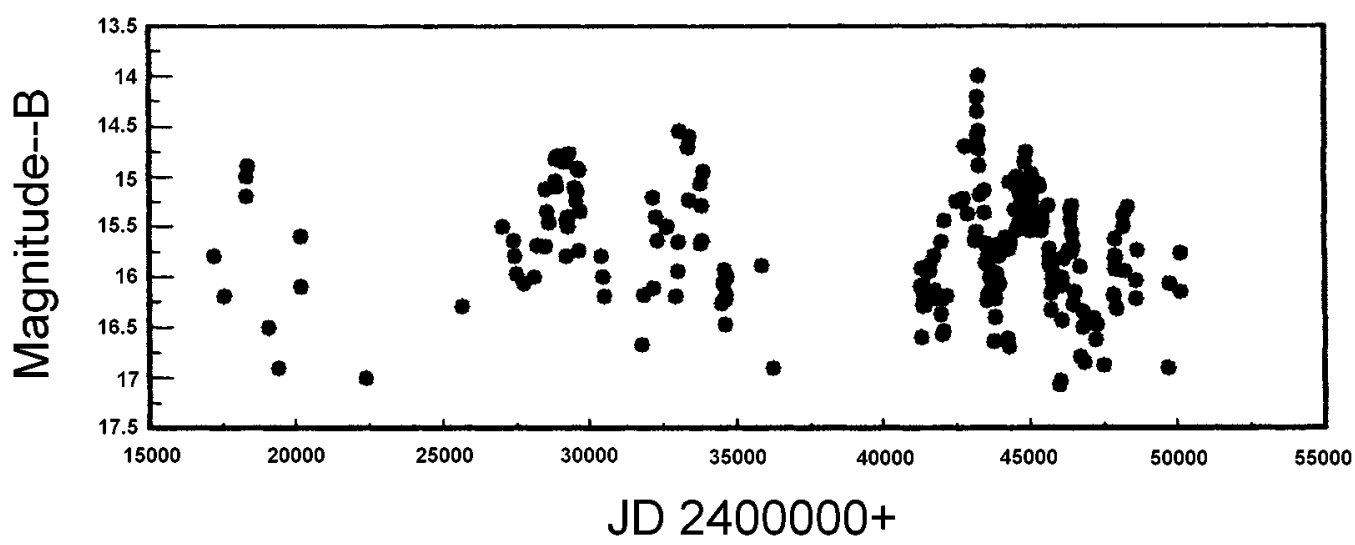

Fig. 2. The mean light curve in the form of 10-day means (218 points)

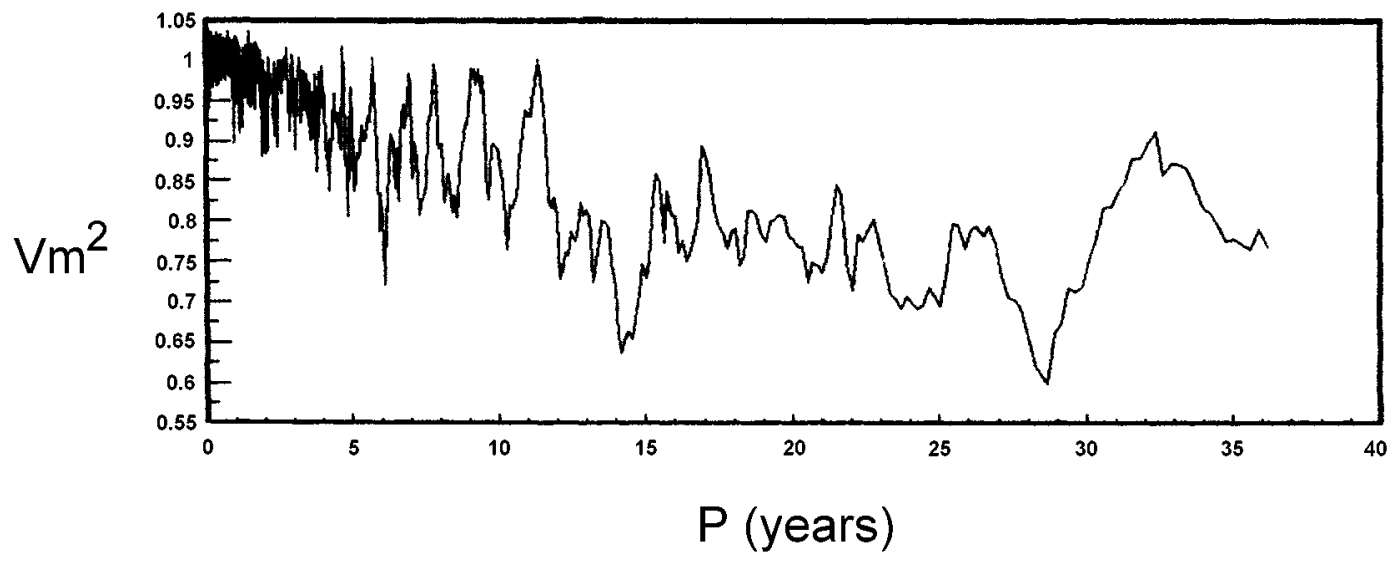

Fig. 3. The normalized Jurkevich test results for the period search for BL Lac object PKS $0735+178$ using the data shown in Fig. 2 with $m=10$ 
are fewer data in the early years than are there in the recent years, the period of 4.89 years can not be ruled out and should be confirmed with more observations.

In this paper, we have shown the light curve of PKS 0735+178 with some new data presented. It is a violent variable object with a large variation of about 4.6 magnitudes and rapid variation of 0.68 magnitude on a time scale of $24 \mathrm{~min}$. The variation is periodic and a period of 14.2 years have been found using Jurkevich method. This kind of long-term period variation may be from the reasons: binary black hole (Sillanpaa et al. 1988) and the thermal and viscous instability in slim accretion disk (Meyer \& Meyer-Hofmeister 1984; Horiuchi \& Kato 1990). In 1991, Valtaoja et al. tried to discuss the variation mechanism in optical band by investigating the correlation of variations in the radio and optical bands. They found that there is no definite correlation of variations in the two bands and proposed that the variation mechanism in the optical band is an unclear mechanism differing from that in the radio band. The period of 4.89 years (Smith et al. 1987a; Webb et al. 1988) can not be ruled out and might be confirmed with more observations, but the period of 1.2 years proposed by them has no sign in our analysis and can be ruled out.

Acknowledgements. We thank an anonymous referee for his valuable comments. This work is supported by the National Natural Science foundation of China and the Natural Science foundation of Guangdong province. We thank Dr. Teresa for her help with the English.

\section{References}

Allen D.A., et al., 1985, ApJS 59, 513

Antonucci R.R.J., Ulvestod J.J.S., 1985, ApJ 294, 158

Baath L.B., Zhang F.J., 1990, in compact steep-spectrum and GHz-peaked spectrum Radio Sources, Fanti C., Fanti R., O'Dea C.P. \& Schilizzi R.T. (eds.) Consighio Nazinale delle Riccerche: Istituto di Radioastronomia-Bologna, p. 147

Bregman J.N., et al., 1984, ApJ 276, 454

Brindle G., et al., 1986, MNRAS 221, 739

Buridge G., Hewitt A., 1987, AJ 92, 1

Carswell R.F., et al., 1974, ApJ 190, L101

Horiuchi T., Kato S., 1990, PASJ 42, 661

Jurkevich I.N., 1971, Ap\&SS 13, 154

Kidger M.R., Yakalo L., Sillanpaa A., 1992, A\&A 264, 32

Kidger M.R., 1989, A\&A 226, 9
Liu F.K., Xie G.Z., Bai J.M., 1995, A\&A 295, 1

Lu P.K., 1972, ApJ 77, 829

McGimsey B.Q., et al., 1975, AJ 80, 895

Meyer F., Meyer-Hofmeister E., 1984, A\&A 132, 143

Miller H.R., 1983, AJ 88, 1301

Moles M., Garcia-Pelayo J.M., Masegosa J., Aparicio A., 1985, ApJS 58, 253

O'Dell S.L., Pushell J.J., Stein W.A., Warner J.W., 1978, ApJS 38, 267

Pollock J.T., et al., 1979, AJ 84, 1658

Pollock J.T., 1975, ApJ 198, L53

Porcas R.W., 1987, in Superluminal Radio Sources, p. 12

Smith A.G., Leacock R.J., Webb, 1987a, Lect. Notes Phys. 307, p. 158

Smith P.S., Balonek T., Elston R., Heckert P.A., 1987, ApJS 64,459

Sillanpaa A., et al., 1996, A\&A 305, L17

Sillanpaa A., et al., 1988a, ApJ 325, 628

Sillanpaa A., et al., 1988b, A\&AS 72, 347

Stevens J.A., Litchfield S.L., Roboson E.I., et al., 1994, ApJ 437, 91

Takalo L.O. Sillanpaa A., Nilsson K., et al., 1992, A\&AS 94, 37

Takalo L.O., 1991, A\&AS 90, 161

Tanzi E.G., et al., 1989, in BL Lac Objects, Maraschi L., Maccacaro T., Ulrich M.-H. (eds.). Springer-Verlag, Heidelberg, p. 171

Terasranta H., et al., 1992, A\&AS 94, 121

Terasranta H, et al., 1991, in Variability of Blazars, Valtaoja E. and Valtonen M. (eds.). Cambridge Univ. Press, p. 159

Ulvestad J.S., Johnston K.J., 1984, AJ 89, 189

Valtaoja L., Karttunen H., Valtaoja E., et al., 1993, A\&A 273, 393

Valtaoja L., Valtaoja E., Shakhovskoy N.M., et al., 1991, AJ 102,1946

Wardle J.F.C., Robert D.H., 1988, IAU 129, p. 143

Webb J.R., Smith A.G., Leacock R.J., et al., 1988, AJ 95, 374

Wing R.F., 1973, AJ 78, 684

Worrall D.M., et al., 1981, ApJ 243, 53

Xie G.Z., et al., 1997 (in preparation)

Xie G.Z., et al., 1994, A\&AS 106, 361

Xie G.Z., et al., 1992, ApJS 80, 683

Xie G.Z., et al., 1990, A\&A 229, 329

Xie G.Z., et al., 1988, A\&AS 72, 163

Xie G.Z., et al., 1987, A\&AS 67, 17

Zekl H., et al., 1981, A\&A 103, 342

Zhang F.J., et al., in Variability of Blazars, Valtaoja E. and Valtonen M. (eds.). Cambridge Univ. Press, p. 234 